\title{
GIST: A propósito de un término confuso y presentación de un caso de tumor del estroma extragastrointestinal
}

\author{
J. A. Contreras Ibañez ${ }^{1}$, L. Atienza Cuevas ${ }^{2}$, J. Pérez Requena ${ }^{2}$, \\ M. Beltrán Ruiz-Henestrosa ${ }^{1}$, J. M. Baena Cañada ${ }^{1}$
}

\section{Resumen}

Propósito: El GIST es un tumor perteneciente a los sarcomas de partes blandas y aunque su localización más frecuente es el tracto gastrointestinal se ha descrito fuera de ésta a pesar de lo cual persiste esa denominación, lo que es, en bastantes casos, motivo de confusión.

Material y métodos: Se presenta el caso de un varón de 57 años diagnosticado, en principio, de "tumor de músculo liso de potencial maligno incierto" en base a su localización retroperitoneal. Tras la revisión y determinación de c-kit (CD-117), se hizo el diagnóstico definitivo de "tumor estromal de localización retroperitoneal”.

Conclusiones: Proponemos el término menos confuso de "tumor estromal con fenotipo de células intersticiales de Cajal".

Palabras clave: GIST. c-kit, Tumores estromales extragastrointestinales. Células intersticiales de Cajal.

Oncología, 2006; 29 (6):254-258

\section{Summary}

Purpose: GIST is a soft tissue sarcoma with preferential location for the gastrointestinal tract. However, it has been described in extragastrointestinal tissues. The persistence of the denomination may eventually lead to confusion, because the abbreviation refers to the gastrointestinal tract

Material and methods: We present the case of a 57 year old man with initial diagnosis of suffering from a "smooth muscle tumor with uncertain malignant potential", based on its retroperitoneal location. After medical examination and c-kit (CD-117) determination, the definitive diagnosis of "stromal tumor of retroperitoneal location" was made.

Conclusions: We propose the term "stromal tumor with Cajal's interstitial cells phenotype".

Key words: GIST. c-kit. Extragastrointestinal stromal tumors. Cajal's interstitial cells.

${ }^{1}$ Servicio de Oncología Médica

${ }^{2}$ Servicio de Anatomía Patológica 


\section{Introducción}

El tumor del estroma gastrointestinal (GIST) es un tumor perteneciente a los sarcomas de partes blandas (SPB) y puede, como todo el grupo, localizarse en cualquier lugar del organismo. Sin embargo, lo más frecuente es que dicha localización sea el tracto gastrointestinal (desde esófago a recto) y de hecho, más del $50 \%$ de los casos lo hacen a nivel gástrico. No obstante, se han descrito en mesenterio y omento ${ }^{1}$, retroperitoneo ${ }^{2}$, vesícula biliar ${ }^{3}$, pared vesical ${ }^{4} \mathrm{y}$ útero ${ }^{5}$.

Su incidencia es baja, estimándose en 10-20 casos por millón de habitantes y año ${ }^{6}$. La edad media de presentación se sitúa entre 50-70 años y muestran una ligera predilección por los hombres. Las manifestaciones clínicas dependen de su tamaño y localización, siendo las más frecuentes el dolor abdominal, la hemorragia digestiva y la masa abdominal, aunque muchos son asintomáticos ${ }^{7}$.

En cuanto a su origen, se postula que derivan de la transformación maligna de las células intersticiales de Cajal (CIC $)^{8-10}$ o de un predecesor más inmaduro y pluripotencial.

Histológicamente se distinguen tres categorías: fusocelular $(70 \%)$, epitelioide $(20 \%)$ y mixta $(5 \%)$, sin que se sepa, hasta el momento, que influencien el pronóstico.

La clave molecular del GIST es la proteína transmembrana KIT (CD117). Al contactar ciertos factores con su porción extracelular, se producen reacciones en cascada que activan su porción intracelular (tirosín-quinasa) con lo que la célula se multiplica con gran rapidez y, además, pierde la capacidad de su muerte programada o apoptosis ${ }^{11,12}$.

Por otra parte, cKit es el gen que codifica dicha proteína. Se localiza en el brazo largo del cromosoma 4 y posee 21 exones. Ya en 1999, se demostró que mutaciones de c-Kit (sobre todo en los exones 11 y 9) daban lugar a formas defectuosas de la proteína KIT y estas mutantes permanecían activadas permanentemente, incluso sin estímulos externos y escapando a los mecanismos de control capaces de "desconectar" la forma normal de la proteína $^{8,13-15}$.

Desde el punto de vista de la inmunohistoquímica (IMH) el 60-70\% de los GIST expresan positividad para CD 34, un 30-40\% son positivos para actina de músculo liso y un 5-10\% son positivos para el antí- geno de la proteína S-100; la desmina es positiva en el 1-2\% de los casos. Sin embargo, ninguno de ellos es específico para diagnosticar GIST en ausencia de positividad para KIT. En 1998, Hirota y cols ${ }^{8}$ comunicaron la expresión de la proteína KIT, pudiendo ser detectada con el anticuerpo monoclonal CD-117. Con ésta técnica, se diagnostican el 94-95\% de los GIST. Heinrich y cols han encontrado mutaciones en el gen PDGFRa en casos de c-Kit negativos y añaden que ambas expresiones son mutuamente excluyentes, pero con consecuencias biológicas similares y sin poder concretar si en el momento actual estos hallazgos tendrían repercusiones pronósticas o terapeúticas ${ }^{15}$. Por otra parte, hay GIST que son ckit negativos y tumores c-kit positivos que no son GIST $^{16}$. Otro marcador inmunohistoquímico que podría ser útil en los c-kit negativos, es la proteína quinasa $\mathrm{C}$ theta (PKC-theta) ${ }^{17}$.

En la Tabla I, se resumen los criterios pronósticos propuestos por Fletcher ${ }^{18}$.

TABLA I

Factores pronósticos en GIST

\begin{tabular}{|lcc|}
\hline Grado de riesgo & Tamaño & $N^{\circ}$ mitosis \\
\hline Muy bajo & $<2 \mathrm{~cm}$ & $<5 \times 50$ CGA \\
Bajo & $2-5 \mathrm{~cm}$ & $<5 \times 50$ CGA \\
Intermedio & $<5 \mathrm{~cm}$ & $6-10 \times 50$ CGA \\
& $5-10 \mathrm{~cm}$ & $<5 \times 50$ CGA \\
Alto & $>5 \mathrm{~cm}$ & $>5 \times 50$ CGA \\
& $>10 \mathrm{~cm}$ & Cualquiera \\
& cualquiera & $>10 \times 50$ CGA \\
\hline
\end{tabular}

CGA: campo de gran aumento

GIST: tumor del estroma gastrointestinal

El tratamiento del GIST resecable es la cirugía completa con bordes amplios y libres; sin embargo a veces no es posible por el gran tamaño de la tumoración. En estos casos, así como en la enfermedad metastásica, el tratamiento es el mesilato de imatinib (Glivec). En la actualidad se están llevando a cabo ensayos clínicos en adyuvancia en pacientes con factores de mal pronóstico que, sin duda, nos permitirán un mejor abordaje de esta enfermedad.

El objetivo del trabajo es intentar esclarecer la, a veces, confusa terminología que rodea a este tipo de neoplasias. 


\section{J. A. Contreras Ibáñez y cols.}

\section{Caso clínico}

Varón de 57 años visto por vez primera en nuestras consultas en marzo de 2003. Fue remitido desde el Servicio de Nefrología tras diagnosticarse de tumor de músculo liso de potencial maligno incierto.

Entre sus antecedentes personales sólo refería cólico nefrítico derecho por el que era estudiado en dicho Servicio al haberse detectado insuficiencia renal. En la TAC se describió una masa abdominal polilobulada de $8 \times 6 \mathrm{~cm}$ que realzaba en parte con contraste y presentaba zonas de necrosis en su interior y que se extendía desde el espacio subfrénico hasta hilio esplénico y cola pancreática.

El 13-2-03 se realizó ecoendoscopia: gran tumoración con áreas de necrosis de probable localización en retroperitoneo, independiente del riñón y de la cola del páncreas que se extendía hacia el polo superior del bazo del que no se podía independizar. No se observaron adenopatías. Se intervino el 6-303 encontrándose tumoración multilobulada entre fornix e hilio esplénico, realizándose extirpación total junto a bazo y pared gástrica a la que se adhiere.

Anatomía Patológica (Figura1): Macroscópicamente y a nivel del hilio esplénico se observa una tumoración multinodular de 11x9x6 cm de consistencia firme y coloración pardusca que al corte presenta frecuentes áreas hemorrágicas. Microscópicamente, el bazo no muestra infiltración tumoral. Fragmento de pared gástrica sin alteraciones. El tu-

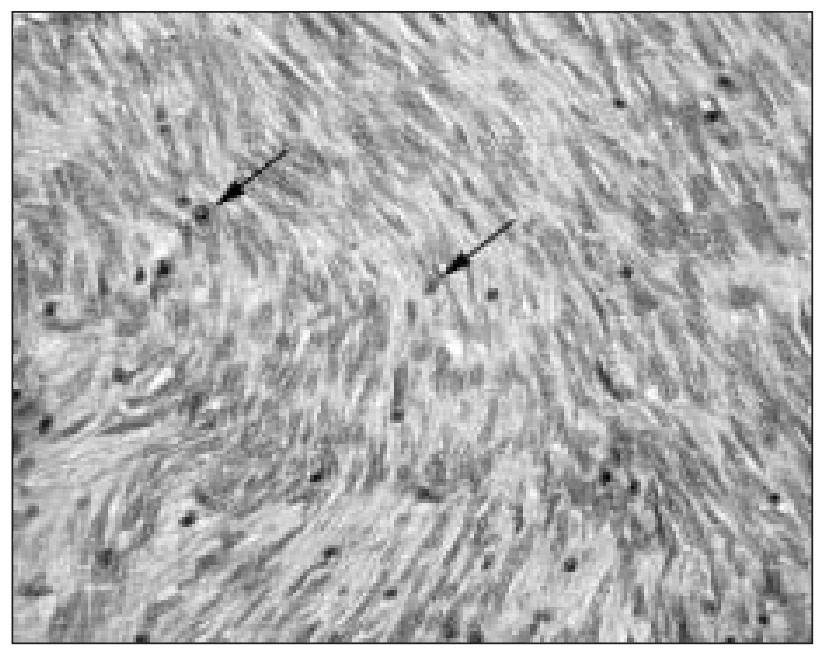

Figura 1. Imagen microscópica del tumor, en la que se observa una población isomorma de células fusiformes que se disponenen en empalizada. Las flechas señalan dos mitosis. (HE x 400).

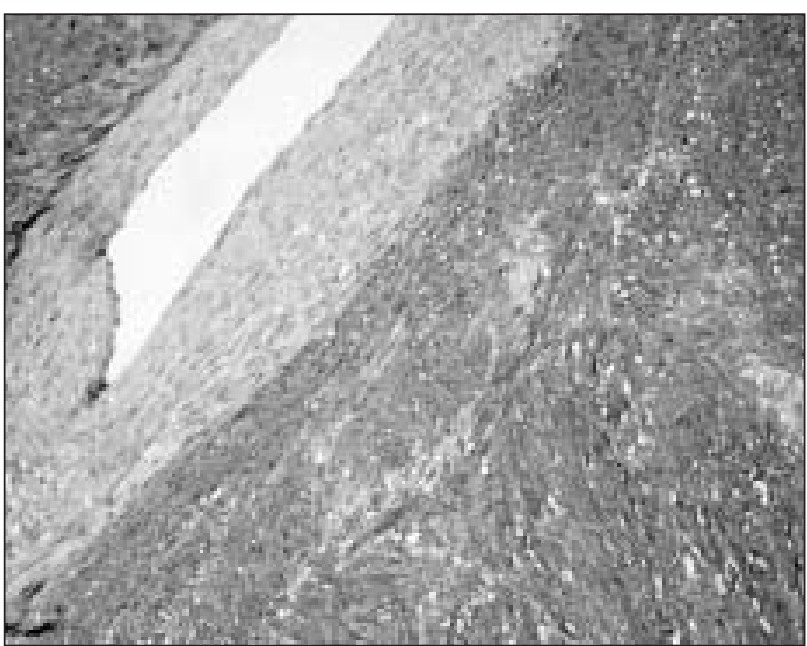

Figura 2. La imagen muestra intensa inmunotinción de c-Kit en las células tumorales. En el centro se observa un vaso sanguíneo sin inmunotinción que sirve como control interno negativo. (Inmunohistoquímica: cKit, x200).

mor descrito previamente está bien delimitado por una delgada cápsula fibrosa y está constituido por células fusiformes isomorfas, sin atipia ni presencia de necrosis, presentado un índice mitótico de $2 \mathrm{mi}-$ tosis por 10 campos de gran aumento. La inmunohistoquímica (IMH) fue positiva para actina de músculo liso y negativa para desmina, S-100, CD 57, EMA, CD68 y neurofilamentos. El diagnóstico fue "Tumor de musculo liso de potencial maligno incierto" (11 cm).

Tras estudio de extensión, que fue negativo, se solicitó una revisión del caso para catalogación definitiva mediante determinación de IMH que en esta ocasión incluyera CD 117; ésta fue intensamente positiva (Figura 2).

Resultados: Actina de músculo liso y Actina muscular específica: inmunotinción débil y focal; CD34 y c-kit (CD 117): inmunotinción intensa con control negativo y positivo para CD 117; S-100 y desmina: ausencia de inmunotinción; Ki67: 10\%.

El diagnóstico definitivo fue tumor estromal de localización en retroperitoneo

\section{Discusión}

Con respecto a los tumores estromales gastrointestinales o GIST, ha existido una variopinta terminología que más que de ayuda ha sido motivo de confusión. Se identificaron en la década de los se- 
senta y se definieron con el término de "leiomioblastomas" 19 . Más tarde se llamaron "sarcomas de fibras musculares lisas" o "leiomiosarcomas" y en 1984 como "plexosarcomas", "GANT" y como "tumores de los elementos neurales de diferenciación neuroendocrina del tubo gastrointestinal no epiteliales" ${ }^{20}$. El descubrimiento de las mutaciones de hiperactivación en el protooncogén c-kit por Hirota y cols en 1998 fue muy importante en la génesis y clasificación de éstos tumores y su tratamiento específico posterior ${ }^{8}$.

Si sabemos en la actualidad que se pueden localizar en zonas extragastrointestinales, ¿por qué seguimos denominándolos "gastrointestinal stromal tumors o GIST?, ¿en cuántas ocasiones han dejado de diagnosticarse por radicar en esas zonas y, por tanto, no ser compatibles con el concepto anatómico?, ¿puede esto haber sido causa de una incidencia subestimada con sus consiguientes implicaciones terapeúticas?

Han sido publicados casos ${ }^{16,21}$ con el término EGIST para hacer referencia a los tumores del estroma de localización extragastrointestinal.

Desde el punto de vista histológico, la situación es complicada y pueden existir errores de diagnóstico por cuanto algunos tumores muestran un patrón mioide o schwanoide, además de expresar arbitrariamente actina de músculo liso, desmina o el antígeno de la proteína S-100. En el caso que presentamos, la inmunohistoquímica fue positiva para actina de músculo liso, dándose como tumor de músculo liso.

En nuestro caso, el tumor estaba constituido por una población de células fusiformes isomorfas sin atipias, que se disponían en fascículos entrecruzados y en empalizada. No se apreciaban focos de necrosis y el índice mitótico era de 2 mitosis por 10 campos de gran aumento. Tras la determinación de CD 117, se catalogó, de tumor estromal retroperitoneal, variedad fusocelular. Como único factor pronóstico adverso tenía el tamaño $(11 \mathrm{~cm})$.

También se han publicado trabajos donde se analiza la expresión Ki-6722, 23 que determinan que si es $>10 \%$ existe mayor riesgo de recidiva o metástasis, aunque quizás el índice sea sólo reflejo del la actividad mitótica. En nuestro caso el Ki-67 fue del 10\% y, como también se ha mencionado, la actividad mitótica baja.

Otros factores como la expresión del factor de crecimiento endotelial vascular (VEGF) ${ }^{24}$, la pérdi- da de expresión de CD $44^{25}$ y las alteraciones de p16 ${ }^{26}$, que se relacionan con peor pronóstico, no fueron analizadas en el caso presentado.

Consideramos, con base en las características del tumor de nuestro paciente y a las indicaciones en esos momentos del uso del Mesilato de Imatinib, un seguimiento periódico del paciente. A los 33 meses de seguimiento, se encuentra libre de enfermedad.

En nuestra humilde opinión, creemos que sería menos confuso el término de "tumores estromales con fenotipo de células de Cajal" con lo que, además, se haría honor a nuestro Premio Nobel.

\author{
Correspondencia: \\ Dr. J. A. Contreras Ibáñez \\ Servicio Oncología Médica, $9^{\text {a }}$ Planta \\ Hospital Universitario Puerta del Mar \\ Avda. Ana de Viya, 21 \\ E-11009 Cádiz \\ jacontreras@auna.com
}

\section{Bibliografía}

1. Suzuki K, Kaneko G, Horigome M. Malignant tumor of the gastrointestinal tumor type, in the greater omentum. $\mathrm{J}$ Gastroenterol 2003; 38(10):958-959.

2. Reith JD, Goldblum JR, Lyles RH, Weiss SW. Extragastrointestinal (soft tissue) stromal tumors: an analysis of 48 cases with emphasis on histologic predictors of outcome: Mod Pathol 2000; 13(5):577-585.

3. Ortiz-Hidalgo C, de León Bojorge B, Albores-Saavedra J. Stromal tumor of the gallbladder with phenotype of intersticial cells of Cajal: a previously unrecognized neoplasm. Am J Surg Pathol 2000; 24(10):1420-1423.

4. Lasota J, Wozniak A, Sarlomo-Rikala M, Rys J, Kordek R, Nassar A et al. Mutations in exons 9 and 13 of KIT gene are rare events in gastrointestinal stromal tumors. A study of 200 cases. Am J Pathol 2000; 157(4):1091-1095.

5. Wingen CB, Pauwels PA, Debiec-Rychter M, Van Gemert WG, Vos MC. Uterine gastrointestinal stromal tumor (GIST). Gynecol Oncol 2005; 97(3):970-972.

6. Kindblom LG. Incidence and prevalence of GIST: practical considerations. En: Glivec, first successful systemic treatment for GIST. London: 2000 (abstract book);18-20.

7. Roberts PJ, Eisenberg B. clinical presentation of gastrointestinal stromal tumors and treatment of operable disease. Eur J Cancer 2002; 38(Suppl 5):537-538. 


\section{J. A. Contreras Ibáñez y cols.}

8. Hirota S, Hisozaki K, Moriyama Y, Hashimoto K, Nishida T. Gain-of-function mutations of c-kit in human gastrointestinal stromal tumors. Science 1998; 279(5350):577-580.

9. Kindblom LG, Remotti HE, Aldenborg F, Meis-Kindblom JM. Gastrointestinal pacemaker cell tumor (GIPACT): gastrointestinal stromal tumors show phenotypic characteristics of the intersticial cells of Cajal. Am J Pathol 1998; 152 (5):1259-1269.

10. Kitamura Y, Hirota S, Nishida T. Gastrointestinal stromal tumors (GIST): A model for molecule-based diagnosis and treatment of solid tumors. Cancer Sci 2003; 94(4):315320 .

11. Pawson T. Regulation and targets of receptor tyrosine kinases. Eur J Cancer 2002; 38(Suppl 5):3-10.

12. Schlessinger J. Signal transduction by allosteric receptor oligomerization. Trends Biochem Sci 1988; 13(11):443447.

13. Rubin BP, Singer S, Tsao C et al. KIT activation is a ubiquitous feature os gastrointestinal stromal tumors. Cancer Res 2001; 61(22):818-821.

14. Rubin BP, Fletcher JA, Fletcher CD. Molecular insights into the histogenesis and pathogenesis of gastrointestinal stromal tumors. Int J surg Pathol 2000; 8 (1): 5-10.

15. Heinrich MC, Corless CL, Duensing A, et al. PDGFRA activating mutations in gastrointestinal stromal tumors. Science 2003; 299 (5607): 708-710.

16. Yamamoto H, Oda Y, Kawaguchi K, et al. C-kit and PDGFRA mutations in extragastrointestinal stromal tumors (gastrointestinal stromal tumors of the sofá tissue). Am J Surg Pathol 2004; 28(4):479-488.

17. Motegi A, Sakurai S, Nakayama H, Sano T, Oyama T, Nakajima T. PKC-theta, a novel immunohistochemical mar- ker for gastrointestinal stromal tumors (GIST), especially useful for identifying KIT-negative tumors. Pathol Int 2005; 55(3):106-112.

18. Fletcher CD. Clinicopathologic correlations in gastrointestinal stromal tumors. Hum Pathol 2002; 33(5):455-456.

19. Stout AP. Bizarre smooth muscle tumors of the stomach. Cancer 1962; 15:400-409.

20. Herrera G. Historical perspective: a jurney through evolution of classification schemes. In USCAP Companion Meeting Syllabus. 2003; 1-6.

21. Nakagawa M, Akasaka Y, Kanai T, Takabayashi T, Miyazawa N. Clinicopathological and immunohistochemical features of extragastrointestinal stromal tumors: report of two cases. Surg Today 2005; 35:(4):336-340.

22. Rudolph P, Peters J, Lorenz D, Schmidt D, Parwaresch R. Correlation between mitotic and $\mathrm{Ki}-67$ labeling indices in paraffin-embedded carcinoma specimen. Hum Pathol 1998; 29(11):1216-1222.

23. Seidal T, Edvardsson H. Expression of c-kit (CD-117) and Ki67 provides information about the possible cell of origin and clinical course of gastrointestinal stromal tumors. Histopathology 1999; 34(5):416-424.

24. Takahashi R, Tanaka S, Kitadai Y, et al. expression of vascular endothelial growth factor and angiogenesis in gastrointestinal stromal tumors of the stomach. Oncology 2003; 64:266-274.

25. Montgomery E, Abraham SC, Fisher C, et al. CD 44 loss in gastric stromal tumors as prognostic marker. Am J Surg Pathol 2004; 28:168-177.

26. Schneider-Stock R, Boltze C, Lasota J, et al. High prognostic value of p16INK4 alterations in gastrointestinal tumors. J Clin Oncol 2003; 21:1688-1697. 